\title{
Alterations in hematologic indices during long-duration spaceflight
}

\author{
Hawley Kunz ${ }^{1}$ (D) Heather Quiriarte ${ }^{2}$, Richard J. Simpson ${ }^{3}$, Robert Ploutz-Snyder ${ }^{4}$, Kathleen McMonigal ${ }^{5}$, \\ Clarence Sams ${ }^{5}$ and Brian Crucian ${ }^{5^{*}}$
}

\begin{abstract}
Background: Although a state of anemia is perceived to be associated with spaceflight, to date a peripheral blood hematologic assessment of red blood cell (RBC) indices has not been performed during long-duration space missions.

Methods: This investigation collected whole blood samples from astronauts participating in up to 6-months orbital spaceflight, and returned those samples (ambient storage) to Earth for analysis. As samples were always collected near undock of a returning vehicle, the delay from collection to analysis never exceeded $48 \mathrm{~h}$. As a subset of a larger immunologic investigation, a complete blood count was performed. A parallel stability study of the effect of a $48 \mathrm{~h}$ delay on these parameters assisted interpretation of the in-flight data.

Results: We report that the RBC and hemoglobin were significantly elevated during flight, both parameters deemed stable through the delay of sample return. Although the stability data showed hematocrit to be mildly elevated at $+48 \mathrm{~h}$, there was an in-flight increase in hematocrit that was $\sim 3$-fold higher in magnitude than the anticipated increase due to the delay in processing.

Conclusions: While susceptible to the possible influence of dehydration or plasma volume alterations, these results suggest astronauts do not develop persistent anemia during spaceflight.
\end{abstract}

Keywords: Spaceflight, Red blood cells, Anemia, Platelets

\section{Background}

A number of physiologic changes are known to occur during prolonged spaceflight. The combined effects of microgravity, radiation, physical and psychological stressors, altered nutrition, disrupted circadian rhythms, and other factors have impacts on many of the body's systems, including vision, the musculoskeletal system, and the immune system [1]. Another marked alteration in physiology is the redistribution of fluids upon entering microgravity, which in turn may influence various hematologic parameters.

Without constant gravitational force, an almost immediate shift of fluids toward the head occurs, resulting in a "puffy" face and a reduced leg volume. An "acute plethora" of blood surrounds the central organs as peripheral blood is no longer held in the extremities by

\footnotetext{
* Correspondence: brian.crucian-1@nasa.gov

${ }^{5}$ NASA Johnson Space Center, 2101 E NASA Parkway, Houston, TX 77058, USA

Full list of author information is available at the end of the article
}

gravity [2-4]. While there is relevant existing information regarding red blood cells and spaceflight, it is primarily associated with short-duration Space Shuttle missions. Hematocrit, red blood cell (RBC) count, hemoglobin and plasma volume have been measured during short-duration spaceflight. RBC count and hemoglobin were found to be elevated throughout a 14-day mission, while plasma volume was found to be decreased $17 \%$ within the first $24 \mathrm{~h}$ immediately after launch, and remained depressed when measured at flight day $8[2,4]$. In the same subjects, RBC mass was measured, but only immediately after landing, at which time a reduction in RBC mass was found [2-4]. The authors ascribed the likely cause of the reduction in $\mathrm{RBC}$ mass to be the "acute plethora" of RBCs resulting from fluid shifts during flight. These reductions in RBC mass following spaceflight have been observed throughout the history of spaceflight $[3,5]$. During 10 to 14 -day space missions, average losses of $10 \%$ to $15 \%$ of RBC mass immediately on landing are consistently reported, corresponding to a loss of approximately $1 \%$ RBC mass per day 
$[3,5]$. These summary alterations result in an approximate $10 \%$ decrease in total blood volume [1] following short duration flight. Similar reductions have been observed in post-flight samples obtained after long-duration spaceflight [3, 5-7]. A reduction in RBC mass during spaceflight, termed "spaceflight anemia," is therefore a generally accepted phenomenon and appears to be a normal adaptation to microgravity $[3,5]$.

A majority of the studies examining alterations in $\mathrm{RBC}$ mass have been limited to post-flight evaluations. The few in-flight evaluations have been limited to short-duration flights, throughout which physiologic adaptations to microgravity are likely to still be occurring. Findings during short-duration flight may therefore not accurately reflect the in-flight condition during long-duration flight. As hematology indices generally do not tolerate freezing and ambient blood samples are rarely returned from space, there is a dearth of in-flight hematologic indices during long-duration spaceflight. The evidence that does exist for long-duration spaceflight appears to indicate that the reductions in RBC mass may actually be less severe for longer missions [7]. Further, very little information regarding the effects of spaceflight on platelets is available [3]. Therefore, additional data describing the in-flight hematologic condition as the body adapts to long-duration spaceflight are needed.

Here we report RBC and platelet indices on blood collected before, during, and after long-duration spaceflight as a subset of a two parent investigations of the effects of long-duration spaceflight on the immune system [8]. Inflight samples were collected in conjunction with crew returns and returned to the laboratory within $48 \mathrm{~h}$, enabling an examination of ambient blood samples collected on board the International Space Station (ISS). A standard complete blood count (CBC) was performed on all samples. Alterations in the bulk leukocyte subsets during spaceflight, including in- and post-flight elevations of white blood cell and granulocyte concentrations, were previously reported alongside additional white blood cell functional data [8]. Here, in-flight hematologic indices were examined in an effort to better understand in-flight alterations in $\mathrm{RBC}$ and platelet parameters during longduration spaceflight. To accurately interpret the data and to determine the impact of the processing delay resulting from the time required to transport ambient blood from the International Space Station (ISS) to the laboratory, a stability study examining the effects of room-temperature blood storage on these indices was also performed.

\section{Methods}

\section{Subjects}

Thirty-one astronaut crewmembers (25 males, 6 females, mean age 52 years, range 38-61) participated in one of two parent investigations, the National Aeronautics and
Space Administration (NASA) 'Integrated Immune' and the University of Houston 'Salivary Markers' studies onboard the ISS. Of the 31 crewmembers, 24 flew on the Russian Soyuz capsule and completed missions of approximately 6 months. The remaining 7 crewmembers rotated to the ISS via the United States Space Shuttle. Of those 7, 5 completed missions lasting greater than 100 days, and 2 had mission durations of less than 60 days.

To determine the effects of room temperature storage on hematologic indices, 20 healthy, adult, non-astronaut subjects (12 males, 8 females, mean age $45 \pm 13$ years, range 26-65) were recruited for a stability study by the NASA Johnson Space Center (JSC) Test Subject Facility. For all astronaut and stability study subjects, approval was obtained from the JSC Institutional Review Board and written informed consent was obtained from all subjects.

\section{Blood sampling}

For both the flight study and the stability study, peripheral blood was collected into a $10.0 \mathrm{~mL}$ ethylenediaminetetraacetic acid (EDTA) spray-coated blood collection tube (BD, Franklin Lakes, NJ, USA). Pre-flight samples were collected at approximately 180 days (L180 ) and 45 days (L-45) prior to launch. In-flight, samples were collected within the first 2 weeks of flight (early), between months 2 and 4 of the mission (mid), and approximately 6 months into the mission, immediately prior to return (late). For those astronauts completing shorter duration missions, only 2 samples were collected and corresponded to the "early" and "mid" time points. Post-flight, samples were collected within 3-8 h post-landing $(\mathrm{R}+0)$ and 30 days post-flight $(\mathrm{R}+30)$. Stability subject samples consisted of a single $10.0 \mathrm{~mL}$ EDTA spray-coated blood collection tube (BD), sampled as indicated following.

\section{Processing}

All CBCs were performed using calibrated, automated hematology analyzers (JSC processing: Coulter LH750, Miami, FL, USA; Kennedy Space Center (KSC) processing: Coulter Gen-S, Miami, FL, USA; Star City, Russia processing: ABX Pentra, Horiba Medical, Irvine, CA, USA; University of Houston Processing: Mindray BC3200, Mindray, Shenzhen, China). Upon arrival to the laboratory, a $1.0 \mathrm{~mL}$ aliquot was removed for $\mathrm{CBC}$ analysis. All pre- and post-flight astronaut blood samples were immediately processed at JSC; however, the analysis of samples collected in-flight was delayed up to $48 \mathrm{~h}$ as a result of the time required to transport the ambient blood from the ISS to the laboratory. Briefly, blood samples were collected from each participating crewmember onboard the ISS (Fig. 1) approximately $10 \mathrm{~h}$ prior to 
hatch closure of the returning vehicle (either Shuttle or Soyuz). Collected blood samples were stored in customized blood pouches and transferred to the returning vehicle for return to Earth. Processing of in-flight samples was performed at JSC or the University of Houston, KSC, or at Star city, Russia, depending on the mission landing site.

To examine the effects of the processing delay on the in-flight samples, sterile syringes were used to obtain $1.0 \mathrm{~mL}$ aliquots from the $10.0 \mathrm{~mL}$ EDTA-coated blood collection tubes collected from healthy donors. The first $\mathrm{CBC}$ was run immediately following blood collection from each stability study subject. Subsequently, blood was stored in the dark at room temperature, and $1.0 \mathrm{~mL}$ aliquots were removed and analyzed at 24,48 , and $72 \mathrm{~h}$ post-collection. All stability samples were processed and analyzed at JSC.

\section{Statistical analysis}

This was a longitudinal, repeated-measures study examining the effects of spaceflight on multiple hematologic parameters. Each astronaut served as his/her own control and all in-flight and post-flight time points were compared to the astronaut's baseline sample. The L-180 time point was considered baseline, as pre-mission stressors may have influenced the L- 45 time point. The distribution of each parameter was tested for normality using the Shapiro-Wilk Normality Test. Non-normal data were transformed logarithmically and outliers were removed for analysis. For all RBC indices, mixed-effects linear models were used to compare each subsequent time point to the L-180 baseline. A random intercept was used to account for the repeated-measures design of the study. Statistical analysis was performed using STATA statistical software (v14, StataCorp LP, College Station, TX, USA). Significance was set at $p<0.05$.

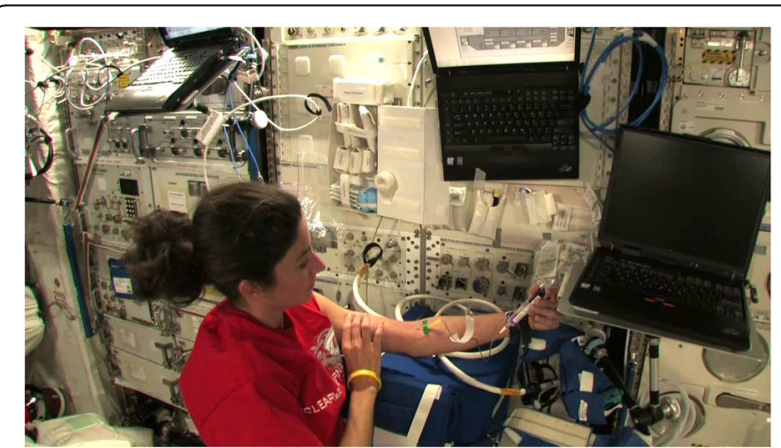

Fig. 1 Blood collection onboard the ISS. Astronaut Nicole Stott performs phlebotomy on the ISS. Samples were collected $\sim 10 \mathrm{~h}$ prior to return vehicle undocking (Space Shuttle or Soyuz). Blood samples were returned to the laboratory for analysis within $48 \mathrm{~h}$ of collection
To determine the stability of the hematologic indices, two one-sided tests of equivalency for dependent samples were performed on the data from the 20 healthy stability study subjects, comparing each of the aged samples to the corresponding Day 0 baseline sample. The within-person coefficient of variation for each hematologic parameter reported by Lacher et al. [9] was used to define the equivalence bounds for the two one-sided tests. Significant results $(p<0.05)$ in the two one-sided tests indicate that the aged samples and the baseline sample are practically equivalent. Results from the stability study were used to inform results from the astronaut study and assist with interpretation, but no direct comparisons were made between the astronauts and the stability study subjects. The stability study statistical calculations were performed using Microsoft Excel and the spreadsheet developed by Lakens [10].

\section{Results}

Of the $\mathrm{RBC}$ and platelet indices included in a $\mathrm{CBC}$, the hematology analyzers measure $\mathrm{RBC}$ count, mean corpuscular volume (MCV), hemoglobin, and platelet concentration. All other parameters are calculated from these measurements. Only the RBC count, hemoglobin, mean corpuscular hemoglobin $(\mathrm{MCH})$, and platelet concentration remained stable for $48 \mathrm{~h}$ at room temperature (Fig. 2a-c, f). Parameters were considered stable if, when compared to the baseline sample, they were significantly within the pre-defined equivalent bounds $(p<0.05)$ at the 24 and $48 \mathrm{~h}$ time points. At both 24 and $48 \mathrm{~h}$ after collection, when compared to the baseline sample, the platelet concentration fell within the pre-defined equivalent bounds $(p<0.05)$; however, at $72 \mathrm{~h}$ after collection, the platelet concentration was no longer significantly practically equivalent to the baseline sample $(\mathrm{t}(19)=-1.554$, $p=0.068)$. Both hematocrit and MCV steadily increased over the $72 \mathrm{~h}$ of storage at room temperature (Fig. $2 \mathrm{~d}$ and e). Compared to baseline, MCV was not within the equivalent bounds at $24 \mathrm{~h}(\mathrm{t}(19)=6.337, p=1.000)$. While elevated at $24 \mathrm{~h}$, hematocrit was significantly within the equivalent bounds $(\mathrm{t}(19)=-1.885, p=0.037)$; however, hematocrit was not significantly within the equivalent bounds by $48 \mathrm{~h}(\mathrm{t}(19)=0.75, p=0.076)$. Given the relationship between hematocrit, $\mathrm{MCV}$, and $\mathrm{RBC}$ count (hematocrit $=[\mathrm{MCV} \times \mathrm{RBC}$ count $] / 10)$ alterations in MCV will necessarily affect the hematocrit values. The elevations in hematocrit over the $72 \mathrm{~h}$ therefore reflect the elevations in MCV. Additional parameters that were measured but not included in any subsequent analysis due to instability following delayed processing include red cell distribution width, mean corpuscular hemoglobin concentration, and mean platelet volume (data not shown).

All astronaut samples drawn on the ISS were returned to the lab within $48 \mathrm{~h}$, and the majority of samples were returned by $\sim 37 \mathrm{~h}$ post-collection. 

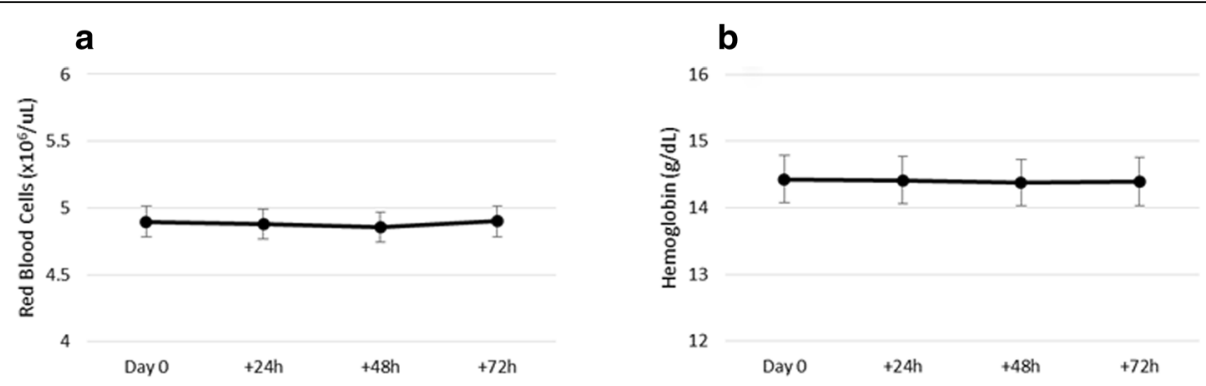

C
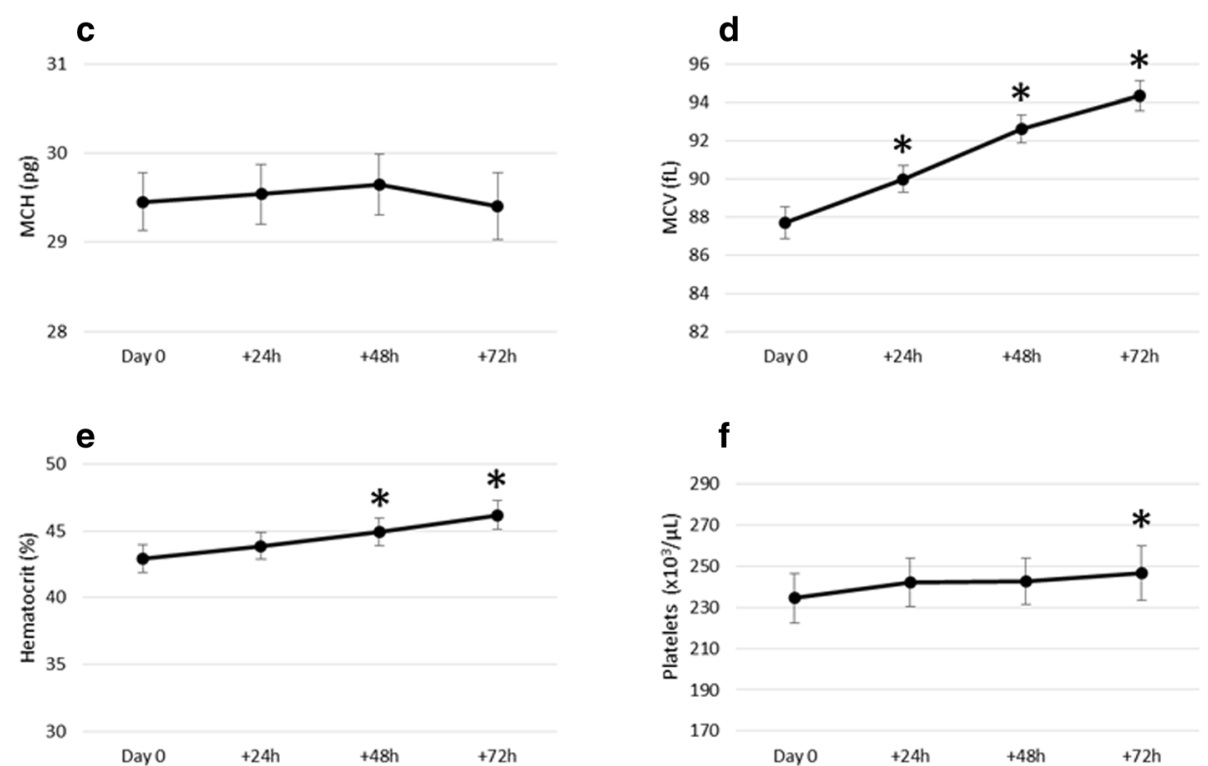

Fig. 2 Hematologic indices evaluated immediately following blood collection, and 24, 48, and $72 \mathrm{~h}$ after collection. All aged samples were compared to the baseline sample analyzed immediately post-collection using two one-sided tests for dependent samples. Data are presented as mean \pm standard error. Samples that were not statistically considered equivalent to the baseline sample $(p>0.05)$ are indicated with *. a Red blood cell concentration $\left(\times 10^{6}\right.$ cells/ $\left.\mathrm{LL}\right) ; \mathbf{b}$ hemoglobin concentration ( $\left.\mathrm{g} / \mathrm{dL}\right) ; \mathbf{c}$ mean corpuscular hemoglobin (MCH; pg); d mean corpuscular volume (MCV; fL); e hematocrit (\%); and $\mathbf{f}$ platelet concentration $\left(\times 10^{3}\right.$ cells $\left./ \mu \mathrm{L}\right)$. All parameters were measured using calibrated automated hematology analyzers

Therefore, only parameters that remained stable at $48 \mathrm{~h}$ were included in the analysis of the effects of longduration spaceflight on hematologic indices, with the exception of hematocrit and MCV, discussed below. The effects of long-duration spaceflight on the analyzed hematologic indices are presented in Fig. 3a-f. All parameters remained consistent prior to flight, with no significant differences between the L-180 and L-60 time points. RBC concentration was significantly elevated at all three in-flight time points compared to the L-180 baseline time point (Fig. 3a; L-180: mean $4.4 \pm 0.4$, range 3.5-5.1; Early: mean $4.8 \pm 0.5$, range 3.9-5.7; Mid: $4.7 \pm 0.4$, range $3.9-5.4$; late: $4.7 \pm 0.4$, range $4.1-$ 5.6). Hemoglobin was elevated early in flight compared to L-180, but returned to pre-flight values as the mission progressed (Fig. 3b; L-180: mean $14.1 \pm 1.4$, range 11.0-17.8; Early: mean $15.0 \pm 1.9$, range 10.7-17.5). Throughout the mission, $\mathrm{MCH}$ decreased, and was significantly lower than the L-180 baseline by the lateflight time point (Fig. 3c; L-180: mean $31.7 \pm 1.6$, range
28.8-36.4; Late: $31.3 \pm 1.9$, range 26.3-34.0). While hemoglobin fell below L-180 baseline values on landing day (Fig. 3b; L-180: mean $14.1 \pm 1.4$, range $11.0-17.8$; $\mathrm{R}+0$ : mean $13.5 \pm 1.4$, range 10.1-15.9), $\mathrm{RBC}$ count and $\mathrm{MCH}$ returned to pre-flight values upon re-entry, and by $\mathrm{R}+30$, all indices were at pre-flight levels.

Significant increases in MCV observed in flight (3.9\%, $4.6 \%$, and $4.2 \%$ increases in mean values compared to the L-180 baseline at early, mid, and late, respectively; Fig. 3d), reflect the changes observed following the $48 \mathrm{~h}$ processing delay $(5.6 \%$ increase in mean values from baseline to +48 h; Fig. 2 d). Therefore, we do not identify any $\mathrm{MCV}$ variations attributable to spaceflight. As noted previously, elevations in MCV will also manifest as elevations in hematocrit. Although hematocrit values increased when subjected to processing delays and were significantly elevated at $48 \mathrm{~h}$ after collection (Fig. 2e), the alterations in hematocrit during spaceflight were striking (Fig. 3e). The significant $(p<0.05)$ elevations in hematocrit observed in flight were of greater magnitude 


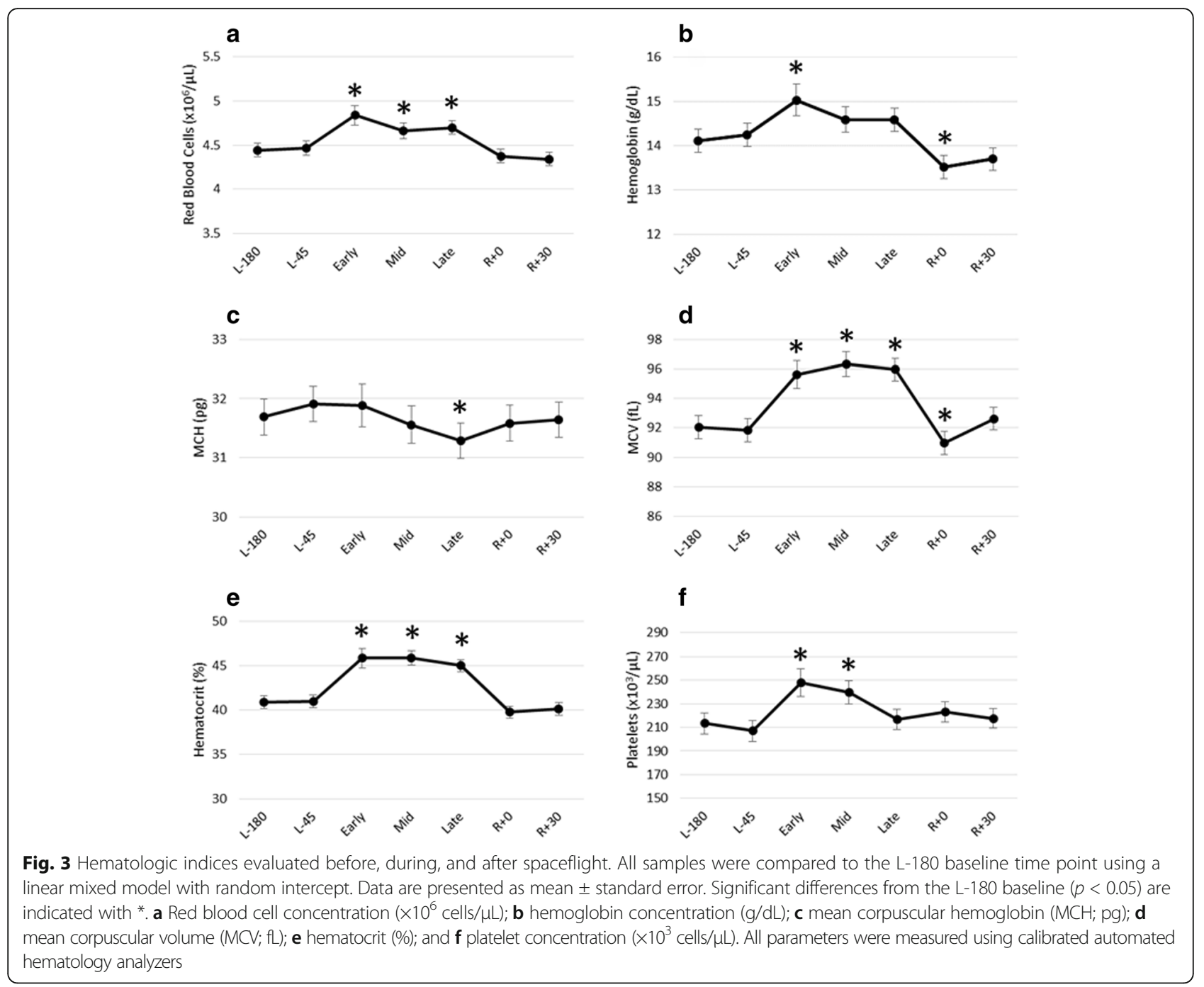

than those observed simply from the elevations in $\mathrm{MCV}$ as a result of the processing delay. A $4.7 \%$ increase in the mean hematocrit was observed after the $48 \mathrm{~h}$ processing delay (Day 0), while the percentage increases in mean hematocrit at the early, mid, and late time points compared to the L-180 time point were $12.2 \%, 12.2 \%$, and $10.0 \%$, respectively (L-180 mean $40.9 \pm 3.9$, range 33.1-48.0; Early: mean $45.9 \pm 4.7$, range $38.2-52.1$; Mid: $45.9 \pm 5.5$, range $38.9-58.3$; Late: $45.0 \pm 2.5$, range $38.9-$ 49.9). These in-flight elevations are therefore most likely due to a combination of a true in-flight increase in RBC count and an artifactual increase in MCV resulting from the processing delay.

Platelet concentration was elevated early in flight. While tracking toward recovery, platelet concentration remained significantly elevated at the mid-flight time point, but was not significantly higher than pre-flight by the late time point (Fig. 3f). The concentration remained stable upon landing and during recovery.

\section{Discussion}

While spaceflight anemia has been consistently reported post-flight and during short-duration flight $[3,5]$, little is known about the in-flight condition during longduration missions. In this study, we observed statistically significant elevations in the concentrations of RBCs, platelets, and hemoglobin, and we interpret an apparent increase in hematocrit at multiple time points during long-duration spaceflight.

The alterations associated with spaceflight observed in this study are in accordance with previous findings of elevated $\mathrm{RBC}$ indices in-flight. RBC concentration, hemoglobin, and hematocrit have been shown to be elevated during the first few days of flight $[2,4,11]$; however, here we show that $\mathrm{RBC}$ concentration remains elevated even after the initial period of adaptation to microgravity. Although previous findings suggest that RBC mass is decreased in association with spaceflight $[2-4,7]$, alterations in cell mass and concentration need 
not track together. While the observed elevations in RBC concentration and hematocrit may simply be due to greater losses in plasma volume than in RBC mass, it is possible that RBC mass is partially restored as the body adjusts to the absence of gravity as flight duration extends, and the losses in RBC mass are less severe during longduration spaceflight. In a review of literature on $\mathrm{RBC}$ mass and spaceflight, Tavassoli et al. [3] noted that in the first 3 weeks of flight, length of flight and losses in RBC mass were positively correlated, with greater losses in RBC mass occurring in longer flights; however, in the studies performed on the longer-duration Skylab 2, 3 and 4 missions (28, 59, and 84 days, respectively), the longer missions were actually associated with smaller decreases in RBC mass $[3,7]$. Therefore it has been previously postulated that during prolonged exposure to microgravity a new $\mathrm{RBC}$ mass homeostasis is reached, and the early reductions in $\mathrm{RBC}$ mass are abrogated $[5,12]$.

The observed reduction in $\mathrm{MCH}$ late in-flight may be reflected in the relationship between $\mathrm{RBC}$ concentration and hemoglobin, as RBC concentration remained elevated throughout the flight while hemoglobin was significantly elevated only early in the flight. A reduced requirement for oxygen-carrying capabilities and easier delivery of oxygen to tissues while in microgravity may drive some of these changes $[5,6]$.

Previous post-flight findings are varied, as both elevations $[7,13]$ and depressions $[7,11]$ in $\mathrm{RBC}$ count, hemoglobin, and hematocrit have been reported. Here we found significant post-flight decreases in hematocrit and $\mathrm{MCV}$, while all other parameters rapidly returned to baseline upon re-entry. Interestingly, immediately after the 28-day Skylab 2 mission RBC count, hemoglobin concentration and hematocrit fell below pre-flight values, and while RBC count had recovered by day 7 post-flight, hematocrit and hemoglobin concentration were still below pre-flight levels at 18 days post-flight [7]. In contrast, on the Skylab 3 and 4 missions (59 and 84 days, respectively) RBC count, hemoglobin concentration, and hematocrit were elevated immediately upon landing, but subsequently began to decline and were significantly lower than pre-flight values 3 days after landing, returning to normal in the 3 week testing period following the flights [7]. With the dependence of these indices on plasma volume, the timing of the sample and the conditions of the return may have a large impact. Both dehydration and plasma volume shifts upon reentry into gravity can significantly affect these parameters. Plasma volume has been shown to be rapidly restored upon re-entry $[14,15]$, which may account for the rapid return to baseline values of $\mathrm{RBC}$ count observed in this study, given the in-flight elevations in these parameters; however, without an accurate measure of plasma volume, it is difficult to make any conclusive statements. Additional sampling between the $\mathrm{R}+0$ and the $R+30$ samples may be beneficial in determining the erythrokinetics post-flight. Depressions in RBC count, hemoglobin concentration, and hematocrit in the weeks after spaceflight were reported after the Skylab missions and by others $[2,7,11,14]$ and were interpreted as potential depressions in red blood cell mass during spaceflight that were slower to recover upon return to Earth than the depressions in plasma volume. Monitoring the $\mathrm{RBC}$ indices in the days following flight in the current study would have provided interesting information, given the observed in-flight elevations, and not depressions, in various hematologic indices.

Little data exist regarding in-flight platelet concentrations [3]; however, the reports that do exist suggest that microgravity and simulated microgravity actually induce a state of thrombocytopenia [16, 17]. In contrast, the elevations in platelet concentration observed in this investigation at the early and mid-flight time points may be due to reductions in plasma volume without any true increase in platelet numbers. The gradual return toward baseline of platelet concentration over the course of the 6-month mission may be indicative of a homeostatic mechanism that serves to counteract elevations in platelet concentration resulting from reduced plasma volume. Interestingly, BE Crucian, SR Zwart, S Mehta, P Uchakin, HD Quiriarte, D Pierson, CF Sams and SM Smith [18] recently reported that plasma thrombopoietin, which stimulates platelet production and is generally elevated when platelet levels are low, was elevated throughout 6-months of orbital spaceflight; however, vascular endothelial growth factor (VEGF) and C-X-C motif chemokine 5 (CXCL5), both of which are platelet-derived and positively correlated with platelet concentration $[19,20]$, were also elevated throughout the 6-month missions [18]. The elevations in plasma VEGF and CXCL5 [16], in conjunction with the finding that platelet concentration was also elevated, appears to indicate that long-duration spaceflight does not induce thrombocytopenia; however, the discrepant finding that thrombopoietin was also elevated [16] warrants further investigation.

Although the performance of a CBC on samples collected during spaceflight generated novel information, these findings must be interpreted with caution. The cellular concentrations are dependent on plasma volume, and therefore the observed elevations may be influenced by reductions in plasma volume without any real increase in cellular mass. Indeed, plasma volume has been shown to decrease by approximately $17 \%$ within the first $24 \mathrm{~h}$ of spaceflight [2]; however, like changes in RBC mass, the alterations in plasma volume have been primarily observed during short-duration flight or postflight, and little evidence exists describing changes in plasma volume during long-duration spaceflight. The 
reductions in plasma volume observed between flight days 8 and 12 by Alfrey et al. [2], while still significant, were smaller than the reductions observed on the first flight day, indicating there may be a continued trend toward plasma volume recovery as time on board the ISS progresses. In a comparison of short and long-duration flights, the average loss in plasma volume for 5 longduration astronauts was marginally lower than the average loss in 29 short-duration astronauts, though this was not statistically significant [21]. To fully interpret the alterations presented in the current study, plasma volume must also be assessed during long-duration space flight.

The measurement of erythropoietin (EPO) in-flight would also aid in the interpretation of the reported findings; unfortunately EPO was not determined as part of the parent immune investigations. EPO controls RBC mass by regulating the rate of division of $\mathrm{RBC}$ progenitors in the bone marrow, and it has also been postulated to play a role in the neocytolysis process by which newly released RBCs are selectively destroyed upon entering into microgravity $[12,15,22]$. EPO has been shown to be reduced early in-flight but elevated following short-duration flight [4], indicating that homeostatic mechanisms attempt to reduce $\mathrm{RBC}$ mass upon entering microgravity and restore it upon landing. However, to our knowledge, EPO has not been measured during long-duration flight. The measurement of EPO in future studies of prolonged spaceflight may help to explain the present findings of elevated RBC count throughout long-duration flight.

The delay in processing for the in-flight blood samples is also a limitation of the study. RBC, hemoglobin and platelet concentration have all been shown to be stable for up to $72 \mathrm{~h}$ when blood samples collected with EDTA are stored at $4{ }^{\circ} \mathrm{C}$ [23]; however, blood samples for our investigations were returned at ambient temperature. Despite the recommendations that samples be refrigerated, results of the stability tests indicate that RBC count, hemoglobin concentration, $\mathrm{MCH}$ values, and platelets remain stable for at least $48 \mathrm{~h}$, even at room temperature. The elevations in hematocrit and $\mathrm{MCV}$ reported here are in accordance with other study findings. MCV begins to increase within 6$12 \mathrm{~h}$ of blood collection, which, in turn, causes an elevation in hematocrit without any alterations in RBC concentration or plasma volume, even in refrigerated samples [23]. While the elevations in hematocrit and MCV hinder our analysis of the in-flight data, the stability of RBC count, hemoglobin, $\mathrm{MCH}$, and platelet concentration over $48 \mathrm{~h}$ indicates that the observed alterations in these parameters are likely caused by factors associated with space-flight, and are not the result of delayed sample processing.

\section{Conclusions}

Spaceflight anemia is a widely reported phenomenon; however, the vast majority of evidence demonstrating reductions in RBC mass has been collected post-flight. To our knowledge, this is one of the first studies to examine hematologic parameters on blood samples collected during long-duration spaceflight. The data suggest that spaceflight anemia may be less of a concern during long-duration spaceflight. However, as previously noted, the fluctuations in these concentration-dependent variables are influenced by changes in plasma volume. Despite this limitation, the sustained elevation of $\mathrm{RBC}$ and platelet concentrations throughout a 6-month mission on board the ISS reported here seems to warrant further investigation, and accurate in-flight assessments of plasma volume during long-duration spaceflight would aid in the interpretation of the findings of this study.

\section{Abbreviations}

CBC: Complete blood count; CXCL5: C-X-C motif chemokine 5; EDTA: Ethylenediaminetetraacetic acid; EPO: Erythropoietin; ISS: International Space Station; JSC: Johnson Space Center; KSC: Kennedy Space Center; MCH: Mean corpuscular hemoglobin; MCV: Mean corpuscular volume; NASA: National Aeronautics and Space Administration; RBC: Red blood cell; VEGF: Vascular endothelial growth factor

\section{Acknowledgements}

The authors thank the ISS crewmembers for participating in this study. The authors also acknowledge the support provided by the JSC Clinical Laboratory, the JSC Mission Integration Team, and the KSC Baseline Data Collection Facility during this study. The authors are particularly grateful for operational support provided by Mimi Shao at KSC and Matt Roper at JSC. The authors also acknowledge discussions with Ms. Jennifer Crucian MT(ASCP), Hematology Department, Methodist St. John Hospital, Houston, Texas, which supported preparation of this manuscript.

\section{Funding}

This work was supported by a grant from the NASA Human Research Program's Human Health and Countermeasures Element to CS/BEC (SMO-015) and NASA grant NNJ10ZSA003N awarded to RJS.

Availability of data and materials

To protect astronaut confidentiality, per NASA policy, all data from this investigation are stored, maintained, and archived in the NASA Life Sciences Data Archive. These data are, with some restrictions, publicly available.

\section{Authors' contributions}

HK performed data analysis and contributed to the writing of the manuscript. $\mathrm{HQ}$ contributed to the data collection. RJS oversaw data collection for ISS astronauts and provided edits to the manuscript. RPS provided assistance with the data analysis and interpretation. KM assisted with data collection, clinical interpretation of the findings and in manuscript development. CS supervised all study procedures, execution of the flight investigations, and manuscript preparation. BEC contributed to data collection for the ISS astronauts, data analysis, and wrote portions of the manuscript. All authors read and approved the final manuscript.

Ethics approval and consent to participate

Study procedures were reviewed and approved by the NASA Johnson Space Center's Institutional Review Board. Written informed consent was obtained from all subjects prior to participation. Ethics approval for all subjects participating in the stability study was obtained under a protocol specific for assay development and validation, which was approved by the NASA Johnson Space Center's Institutional Review Board.

Consent for publication

The astronaut photographed in Fig. 1 reviewed the image and provided written consent to publish. 


\section{Competing interests}

The authors declare that they have no competing interests.

\section{Publisher's Note}

Springer Nature remains neutral with regard to jurisdictional claims in published maps and institutional affiliations.

\section{Author details}

${ }^{1}$ KBRwyle, 2400 NASA Parkway, Houston, TX 77058, USA. ${ }^{2}$ Louisiana State University, Baton Rouge, Louisiana 70803, USA. ${ }^{3}$ University of Houston, 4800 Calhoun Rd, Houston, TX 77004, USA. ${ }^{4}$ University of Michigan School of Nursing, 400 North Ingalls Building, Ann Arbor, MI 48109, USA. ${ }^{5}$ NASA Johnson Space Center, 2101 E NASA Parkway, Houston, TX 77058, USA.

Received: 20 January 2017 Accepted: 8 August 2017

Published online: 08 September 2017

\section{References}

1. Williams D, Kuipers A, Mukai C, Thirsk R. Acclimation during space flight: effects on human physiology. Can Med Assoc J. 2009;180(13):1317-23.

2. Alfrey CP, Udden MM, Leach-Huntoon C, Driscoll T, Pickett MH. Control of red blood cell mass in spaceflight. J Appl Physiol. 1996;81(1):98-104

3. Tavassoli M. Anemia of spaceflight. Blood. 1982;60(5):1059-67.

4. Udden MM, Driscoll TB, Pickett MH, Leach-Huntoon CS, Alfrey CP. Decreased production of red blood cells in human subjects exposed to microgravity. J Lab Clin Med. 1995;125(4):442-9.

5. Smith SM. Red blood cell and iron metabolism during space flight. Nutrition. 2002;18(10):864-6.

6. De Santo NG, Cirillo M, Kirsch KA, Correale G, Drummer C, FrassI W, Perna AF, Di Stazio E, Bellini L, Gunga HC. Anemia and erythropoietin in space flights. Semin Nephrol. 2005;25(6):379-87.

7. Kimzey SL. Hematology and immunology studies. In: Johnston RS, Dietlein LF editors. Biomedical results from Skylab. Washington, D.C.: NASA; 1977. p. 249-82.

8. Crucian BE, Stowe RP, Mehta SK, Quiriarte H, Pierson DL, Sams CF. Alterations in adaptive immunity persist during long-duration spaceflight. Npj. Microgravity. 2015;2015:1.

9. Lacher DA, Barletta J, Hughes JP. Biological variation of hematology tests based on the 1999-2002 National Health and nutrition examination survey, In: National Health Statistics Reports: Centers for Disease Control and Prevention, vol. 2012. p. 54.

10. Lakens D. Equivalence tests: a practical primer for $\mathrm{t}$ tests, correlations, and meta-analyses. Soc Psychol Personal Sci. 2017; doi:10.1177/ 1948550617697177.

11. Leach CS, Johnson PC. Influence of spaceflight on erythrokinetics in man. Science. 1984;225(4658):216-8.

12. Risso A, Ciana A, Achilli C, Antonutto G, Minetti G. Neocytolysis: none, one or many? A reappraisal and future perspectives Front Physiol. 2014;5:54.

13. Leach CS. Biochemical and hematologic changes after short-term space flight. Microgravity Q. 1992;2(2):69-75.

14. Alfrey CP. The influence of space flight on erythrokinetics in man, Space life sciences missions 1 and 2. Experiment E261 final report. Washington, D.C.: NASA; 1995

15. Alfrey CP, Udden MM, Huntoon CL, Driscoll T. Destruction of newly released red blood cells in space flight. Med Sci Sports Exerc. 1996;28(10 Suppl):S42-4

16. Davis TA, Wiesmann W, Kidwell W, Cannon T, Kerns L, Serke C, Delaplaine T, Pranger A, Lee KP. Effect of spaceflight on human stem cell hematopoiesis: suppression of erythropoiesis and myelopoiesis. J Leukoc Biol. 1996;60(1):69-76.

17. Kalandarova MP. Changes in hematologic indicators in personnel testing during 370-day anti-orthostatic hypokinesia. Kosm Biol Aviak Med. 1991; 25(3):15-8.

18. Crucian BE, Zwart SR, Mehta S, Uchakin P, Quiriarte HD, Pierson D, Sams CF, Smith SM. Plasma cytokine concentrations indicate that in vivo hormonal regulation of immunity is altered during long-duration spaceflight. J Interf Cytokine Res. 2014;34(10):778-86.

19. Feng $X$, Scheinberg P, Samsel L, Rios O, Chen J, McCoy JP Jr, Ghanima W, Bussel JB, Young NS. Decreased plasma cytokines are associated with low platelet counts in aplastic anemia and immune thrombocytopenic purpura. J Thromb Haemost. 2012;10(8):1616-23.

20. Gunsilius E, Petzer AL, Gastl G. Space flight and growth factors. Lancet. 1999; 353(9163):1529.
21. Meck JV, Reyes CJ, Perez SA, Goldberger AL, Ziegler MG. Marked exacerbation of orthostatic intolerance after long- vs. short-duration spaceflight in veteran astronauts. Psychosom Med. 2001;63(6):865-73.

22. Alfrey CP, Rice L, Udden MM, Driscoll TB. Neocytolysis: physiological downregulator of red-cell mass. Lancet. 1997;349(9062):1389-90.

23. Zini G. International Council for Standardization in H. Stability of complete blood count parameters with storage: toward defined specifications for different diagnostic applications. Int J Lab Hematol. 2014;36(2):111-3.

\section{Submit your next manuscript to BioMed Central and we will help you at every step:}

- We accept pre-submission inquiries

- Our selector tool helps you to find the most relevant journal

- We provide round the clock customer support

- Convenient online submission

- Thorough peer review

- Inclusion in PubMed and all major indexing services

- Maximum visibility for your research

Submit your manuscript at www.biomedcentral.com/submit 